\title{
TRANSPORTE DE MINERAL POR MEDIO DE FAJAS TRANSPORTADORAS EN UNA MINA A TAJO ABIERTO
}

\author{
Responsable : MSc. Carlos Huisa Ccori \\ Miembros : Ing. Gualberto Tejada Bedoya \\ Ing. Zenón Sarmiento Mejia
}

\section{RESUMEN}

Uno de los problemas álgidos que no permite a las empresas cumplir su misión y visión de negocio es el aumento en los precios del petróleo y sus derivados, el problema de la inflación y el incremento en los costos de mantenimiento; y el consumo de combustibles para el transporte del mineral de mina hace imprescindible la búsqueda de nuevas alternativas de transporte comoserlas fajas transportadoras.

El objetivo de este trabajo es buscar una alternativa para disminuir los costos de transporte por medio de fajas transportadoras. Según el análisis, se llegó a concluir que existe un ahorro entre faja-volquetes en $0,135 \$$ /t y entre trenes - fajas en $0,066 \$$ th.

Según la evaluación económica, se llego a los siguientes resultados : TIR (183\%), VPN a $12 \%(6,74 \mathrm{M} \$)$, VPN a $15 \%$ $(5,95 \mathrm{M} \$)$. Con un periodo de recuperación de 1,5 años. Por estos resultados consideramos muy tentativa la aplicación del proyecto en las Empresas Mineras.

\begin{abstract}
One of the algid problems that do not allow the companies to fulfill its mission and vision of business are the increase in the prices of petroleum and their derivatives, the problem of the inflation and the increase in the maintenance costs; and the fuel consumption for the transport of the mineral of mine, makes impresendible the search of new alternatives of transport as to be The transporting strips.
\end{abstract}

The objective of this work is to look for an alternative of desminuir the costs of transport by means of transporting strips. According to the analysis I get myself to conclude that a saving between strip exists - dumps in $0.135 \$ / t$. and between trains - strips in $0.066 \$ / t$.

According to the economic evaluation I reach the following results: TIR (183\%), VPN to $12 \%$ (6,74 Ms \$), VPN to $15 \%(5,95$ Ms $\$$ ). With a period of recovery of 1,5 years. By these results we considered the application of the project in the Mining Companies very tentative.

\section{INTRODUCCIÓN}

En la explotación de una mina a tajo abierto, se realiza el planeamiento referente al minado de las reservas económicas mediante el sistema (volquetes - tren). Pero el número de equipos necesarios por año es entonces calculado como producto de la simulación.

Realmente, muchas características del diseño del plan de minado se ven afectados si el numero de equipos es insuficiente. En este caso se debe tratar el diseño de botaderos cortos, cambiar el plan de minado en zonas altas 0 diseñar rampas que permitan el acceso de trenes a zonas de volquetes, etc. Las soluciones anteriores son paliativos a la falta de volquetes, en caso de que esta deficiencia se repita en toda la vida de la mina debe pensarse en inversión.

El análisis económico debe intervenir para decidir si se compran volquetes adicionales, trenes 0 se introducen nuevas alternativas de transporte como las fajas transportadoras.

\section{OBJETIVO}

El ahorro en costo operativo llevado a un flujo económico debe ser superior a la inversión por el sistema de chancado- fajas, de las inversiones programadas por la renovación del sistema actual de volquetes, tolvas y trenes, de tal forma que llevado dicho flujo a una tasa de descuento determinada, se debe obtener una rentabilidad superior.

\section{TRANSPORTE DE MINERAL POR MEDIO DE FAJAS TRANSPORTADORAS}

1) Fajas Transportadoras y sus Aplicaciones: Una de las alternativas es el transporte de mineral de mina a través de fajas transportadoras, sistema que presenta grandes ventajas con respecto al transporte convencional, algunas de las cuales describimos a continuación:

- El transporte de material puede tener pendientes de $+/-30 \%$, con mejor eficiencia que camiones y trenes.

- Las fajas pueden ser instaladas en diferentes niveles, ser movibles y adecuarse a las necesidades de operación.

- Puede cruzar carreteras, rampas, líneas de tren y otras obstrucciones a través de puentes y soportes.

- Puedentener grandes longitudes.

- Tienen una relativa uniformidad en la demanda de energia y electricidad.

- Tienen alta disponibilidad y el mantenimiento se 
reduce a costos minimos.

- Ayuda a mantener los costos de operación pese a la inflación y aumento en los costos de combustible.

- Tienen gran capacidad y con chancadoras primarias en el tajo, pueden trasladarse según las necesidades de operación.

- Actualmente en el mercado hay chancadoras para cada necesidad particular.

Entre algunas desventajas o limitaciones, podemos mencionar:

- El diámetro del material está limitado a 8" y como máximo a $12 "$, por lo que es necesario una parrilla o chancadora en el tajo.

- Los costos de mantenimiento son mayores cuando las dimensiones de los bloques de material son grandes y hay bastante agua.

\section{2) Diseño del proyecto de Transporte por Faja en la Mina.}

- Sistema Camión-Chancado-Faja

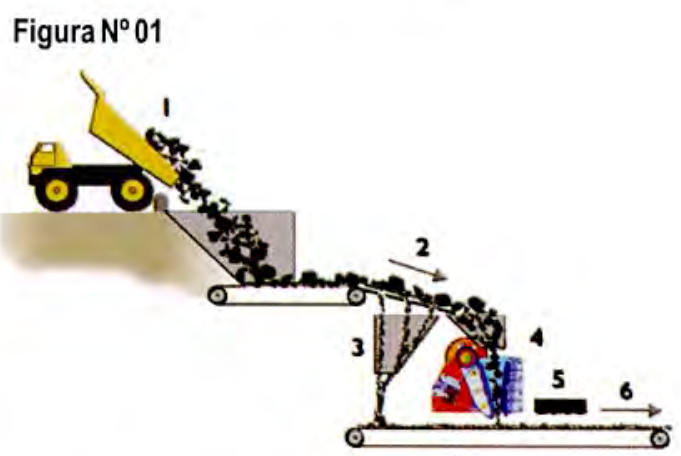

\section{3) Sistema de Transporte de Mineral por Medio de Fajas Transportadoras Propuesto por "Continental Conveyor"}

\section{Datos Básicos:}

- Material a transportar: Mineral de cobre, chancado, no clasificado por tamaño.

- Grueso: máximo tamaño es 300 mm. (abertura nominal de la chancadora primaria), gruesos y pesados con filos cortantes.

- Densidad: $2,54 \mathrm{t} / \mathrm{m}^{3}$ (promedio)

- Factor de esponjamiento: $25 \%$

- Densidad aparente: $2,54 /(1+0,25)=2,0 \mathrm{t}^{3}$.

- Tonelaje por año: 110 millones de toneladas al año.

- Condiciones de operación: 20 horas por dia, 6 dias por semana.

- Ángulo de reposo del material sobre la faja: $15 \%$

- Humedad: $8 \%$

- Abrasividad: media.

\section{Características de la Faja Transportadora}

- Velocidad de la faja: Para transportar mineral de cobre, la velocidad recomendada deberá ser de $3 \mathrm{~m} / \mathrm{s}$. Ancho de faja: Seleccionamos un ancho de faja de $1600 \mathrm{~mm}$. Diseño de polines: Los polines portantes (los que soportan la faja con carga), serán tres (3 - part.), dispuestos en forma de anales, haciendo los polines inclinados un ángulo de $20^{\circ}$ con respecto a la horizontal, manteniéndose el polin central en forma horizontal. La longitud de cad polin será de 600 $\mathrm{mm}$.

Los polines de retorno serán horizontales, con una longitud de $1800 \mathrm{~mm}$. De tal manera que la faja se mantenga plana en el tramo de retorno.

- Diámetro de polines:

Portante: $159 \mathrm{~mm}$

Retorno: $133 \mathrm{~mm}$.

Impacto: $159 \mathrm{~mm}$.

- Peso de polines:

Portante: $42,4 \mathrm{~kg}$

Retorno: $27,6 \mathrm{~kg}$.

- Espaciamiento entre polines:

$L_{0}=5(k)-0.2$

Donde:

$k=$ máx. tam. del bloque $=300 \mathrm{~mm}$.

Porlo tanto: $\mathrm{L}_{0}=1,59 \mathrm{~m}$.

El fabricante recomienda: $1,0 \mathrm{~m}$.

Espaciamiento entre polines de retorno (Lu)

$L u=2,5$. Lo

$L u=2,5 \times 1,0 \mathrm{~m} .=2,5 \mathrm{~m}$

- Masa de polines

El fabricante recomienda utilizar lo siguiente para hallar la masa de los polines:

$M p=M p o / L o+M p u / L u$

Donde:

$M p=$ masa de polines $(\mathrm{kg} / \mathrm{m})$

$\mathrm{Mpo}=$ peso polines portadores $=42,4 \mathrm{~kg} / \mathrm{m}$.

$\mathrm{Mpu}=$ peso polines de retorno $=27,6 \mathrm{~kg} / \mathrm{m}$.

$\mathrm{Lo}=$ espaciam. entre polines portantes $=1,0 \mathrm{~m}$

$\mathrm{Lu}=$ espaciam. entre polines de retorno $=2,5 \mathrm{~m}$

Entonces:

$M p=42,4 / 1,0+27,6 / 2,5$

$M p=53,4 \mathrm{~kg} / \mathrm{m}$

\section{Selección de la Faja Transportadora}

Se utilizará para el transporte de mineral por medio de fajas transportadoras en mina, una faja compuesta por un núcleo formado por cables de acero, que resisten a la tracción, y de dos capas de goma de distintas características, que protegen a dicho núcleo. 
La faja a utilizarse tendrá las siguientes caracteristicas:

\section{$1800-$ St $3500-10+7 M$}

Donde:

1800: Ancho de la faja en $\mathrm{mm}$.

St : Núcleo resistente formado por cables de acero

3500: Resistencia a la rotura del núcleo en sentido longitudinal en $\mathrm{kg} / \mathrm{cm}$

10 : Espesor del recubrimiento superior de goma en mm.

7 : Espesor del recubrimiento inferior de goma en $\mathrm{mm}$.

M : Grado de calidad de goma de recubrimientos

Peso de la faja:

$\mathrm{Mf}=40 \mathrm{~kg} / \mathrm{m}^{2} \times$ ancho faja $(\mathrm{m})$

$M f=40 \times 1,6=64 \mathrm{~kg} / \mathrm{m}$

Masa del mineral de cobre:

$\mathrm{Mn}=\mathrm{Qm} /(3,6 . v)$

$\mathrm{Mn}=3750 /(3,6 \times 3)$

$\mathrm{Mn}=347 \mathrm{~kg} / \mathrm{m}$

Resistencia al movimiento de la faja a utilizarse en Mina.

Resistencia primaria (Fh)

$F h=f \cdot L \cdot\{M p+(2 M f+M h) \cdot \cos \} \cdot G$

Resistencia secundaria(Fn)

$\mathrm{Fn}=(\mathrm{C}-1) \cdot \mathrm{Fh}$

Resistencia a la gradiente $(\mathrm{Fg})$

$$
\begin{aligned}
& \mathrm{Fg}=\mathrm{H} \cdot \mathrm{Mn} \cdot \mathrm{g} \\
& \text { Donde: } \\
& L=\text { Longitud del Transportador } \\
& \mathrm{H}=\text { Altura del Transportador } \\
& f=\text { Chef.de R resistencia } \\
& \mathrm{C}=\text { Chef. de longitud } \\
& \text { Mp = Masadepolin } \\
& 53,4 \mathrm{~kg} / \mathrm{m} \\
& \text { Mf = Masa delaFaja } \\
& 64,0 \mathrm{~kg} / \mathrm{m} \\
& \text { Mn = Masa de la Carga } \\
& 347 \mathrm{~kg} / \mathrm{m} \\
& \mathrm{g}=\text { Gravedad } \\
& 9,8 \mathrm{~m} / \mathrm{s}^{2}
\end{aligned}
$$

4) Transporte de Mineral de Cobre por fajas Desde el Nv. 3335 hasta el Nv. 3427. (Primera Etapa)

$\begin{array}{lll}\text { Longitud del Transp. } & : & L=350 \mathrm{~m} \\ \text { Altura del Transp. } & : & H=92 \mathrm{~m} .\end{array}$

$\begin{array}{lll}\text { Inclinación } & : & \theta=15^{\circ} \\ \text { Coeficiente de resist. } & : & f=0.017 \\ \text { Coeficiente de longitud } & : & C=1.27\end{array}$

\section{Resistencia Primaria}

$$
\begin{aligned}
& F h=f . L .\{M p+(2 M f+M n) \operatorname{Cos} \theta\} g \\
& F h=0,017 \times 350\{53,4+(2 \times 64+347) \cdot \cos \\
& \left.15^{\circ}\right\} \times 9,8 \\
& F h=29867 \text { Newton }
\end{aligned}
$$

\section{Resistencia Secundaria}

$$
\begin{aligned}
& F_{n}=(C-1) \cdot F h \\
& F_{n}=(1,27-1) \times 29867 \\
& F_{n}=8064 \text { Newton }
\end{aligned}
$$

\section{Resistencia a la Gradiente}

$$
\begin{aligned}
& \mathrm{Fg}=\mathrm{H} \cdot \mathrm{Mn} \cdot \mathrm{g} \\
& \mathrm{Fg}=92 \times 347 \times 9,8 \\
& \mathrm{Fg}=312855 \text { Newton }
\end{aligned}
$$

\section{Resistencia total al Movimiento de la Faja}

$$
\begin{aligned}
& \mathrm{Ft}=\mathrm{Fh}+\mathrm{Fn}+\mathrm{Fg} \\
& \mathrm{Ft}=29867+8064+312855 \\
& \mathrm{Ft}=350786 \text { Newton }
\end{aligned}
$$

\section{Tensiones de la Faja Transportadora - Tensión} efectiva (Te)

$$
\mathrm{Te}=\mathrm{Ft}=350786 \text { Newton }
$$

\section{Tensión del Lado Flojo $\left(\mathrm{T}_{2}\right)$}

$$
\begin{aligned}
& T_{2}=T e \cdot C \\
& \begin{array}{l}
\text { Donde } \\
C=0,084 \text { (factor de transmisión) } \\
T_{2}=350786 \times 0,084 \\
T_{2}=29466 \text { Newton }
\end{array}
\end{aligned}
$$

\section{Tensión del Lado Tenso $\left(T_{1}\right)$}

$$
\begin{aligned}
& T_{1}=T e+T_{2} \\
& T_{1}=350786+29466 \\
& T_{1}=380252 \text { Newton }
\end{aligned}
$$

\section{Tensión de Operación unitaria (Tu)}

$$
\begin{aligned}
& T u=T_{1} / \text { ancho faja }(\mathrm{mm}) \\
& T u=380252 / 1600 \\
& T u=238 \mathrm{~N} / \mathrm{mm}
\end{aligned}
$$

\section{Potencia del Sistema de Transmisión}

Potencia requerida

$$
\begin{aligned}
& P=(\text { Te } . v) / 1000 \\
& P=(350876 \times 3) / 1000 \\
& P=1052 \mathrm{kw}
\end{aligned}
$$




\section{Potencia total requerida}

$$
\begin{aligned}
& \mathrm{Pt}=1052+10 \% \\
& \mathrm{Pt}=1157 \mathrm{kw} \\
& \mathrm{Pt}=1551 \mathrm{HP}(1 \mathrm{HP}=0,746 \mathrm{kw}) \\
& \text { Consideremos } \mathrm{Pt}=1600 \mathrm{HP} .
\end{aligned}
$$

Motor principal (polea primaria)

$\mathrm{Pp}=1000 \mathrm{HP}$

Motor secundario (polea secundaria)

$\mathrm{PS}_{\mathrm{S}}=600 \mathrm{HP}$.

Consumo de energia eléctrica

$\left(\right.$ Etapa $\left.N^{0} 01\right)=1157 \mathrm{kw} /$

\section{SISTEMA DE TRANSPORTE POR FAJA PROPUESTAY COSTOS}

\section{1) Análisis de Costo del Uso de Faja Transportadora en Mina}

El sistema móvil de chancado / transporte por faja dentro de mina operará durante 24 años a una tasa de producción de mineral de $140000 \mathrm{tms} / \mathrm{dia}$. En el Plan se han considerado levantamientos nominales de 10 bancos; es decir, un alzamiento máximo de $150 \mathrm{~m}$ para una faja con pendiente máx. de $30 \%\left(16,7^{\circ}\right)$.

Según esta evaluación, el ahorro total en el costo de operación de acarreo por volquetes para los 24 años es de US\$111 millones. Esto se traduce en un ahorro en el costo de minado de mineral de $\$ 0,058 / \mathrm{TM}$ durante los primeros 12 años de funcionamiento (2008-2019) y de $\$ 0,135 / T M$ durante los últimos 12 años de funcionamiento (2020-2031). Los ahorros más importantes ocurren en los últimos años de la vida de mina cuando la fuente del mineral extraido está ubicada en los niveles más profundos. Ver figura $\mathrm{N}^{\circ} 4.1$.

Cuadro $N^{0}$ 4.1: Resultados económicos comparativos entre fajas y volquete

\begin{tabular}{|l|c|c|c|c|}
\hline \multicolumn{1}{|c|}{ Alternativa } & $\begin{array}{c}\text { Ingreso } \\
\text { (M \$) }\end{array}$ & $\begin{array}{c}\text { Costo de } \\
\text { operación (M S) }\end{array}$ & $\begin{array}{c}\text { Utilidad antes de } \\
\text { impuesto (M \$) }\end{array}$ & $\begin{array}{c}\text { Utilidad } \\
\text { descontada (M S) }\end{array}$ \\
\hline FAJAS & $13,693,268$ & $9,537,195$ & $4,156,073$ & $1,244,788$ \\
\hline VOLQUETES & $13,700,319$ & $9,648,004$ & $4,052,315$ & $1,231,232$ \\
\hline
\end{tabular}

\section{2) Evaluación Económica}

Para la evaluación de las fajas transportadoras. Y trenes tenemos lo siguiente:

Cuadro $N^{\circ}$ 4.2: Montos de Inversión y Costo Operativo

\begin{tabular}{lcr} 
TRENES & P.Unt(M \$) & Tot \\
(5) locomotoras & 4.0 & 20.0 \\
.120 vagones & 0.17 & 19.92 \\
. Cambio líneas Ferr. & 0.50 & 3.75 \\
. Reubicación de Hooper & 0.25 & 0.25 \\
& Total $=51.92 \mathrm{M} \$$ \\
\multicolumn{2}{c}{ Costo Operativo $($ S/t) $=$} & 0.147 \\
FAJAS & \\
Chancadora 8164 tmph & $12,5(\mathrm{M} \$)$ \\
$.7500 \mathrm{~m}$. Faja & 18,3
\end{tabular}
Desaduanaje (12\%)
3,7
Instalación (25\%)
8.0
. Total
42,5
Costo Operativo (\$/t)
0,080
Capital asumido $(+25 \%)$
$53.1 \mathrm{MS}$
Costo Operativo $(+35 \%)$
$0,108 \mathrm{~S} / \mathrm{t}$

Con esta información llevado a una secuencia anual de minado, se conformó un flujo económico, los resultados de dicha evaluación económica fueron los siguientes:

$$
\begin{array}{ll}
\text { TIR } & =183 \% \\
\text { VPNaTD a } 12 \% & =6.74 \mathrm{MS} \\
\text { VPNa TD a } 15 \% & =5.95 \mathrm{MS}
\end{array}
$$

Periodo Retorno de la Inversión $=1.5$ años

Como se aprecia, los evaluadores económicos arrojan muy buenos resultados a favor del sistema de fajas, lo cual ya se preveia, ya que el costo de inversión para ambas alternativas es muy similar, siendo el costo operativo del sistema de fajas, prácticamente la mitad del sistema de acarreo por trenes.

El tiempo para tomar la decisión e implementación es un factor importante, debido a que en el año 2007 se mina una de las tolvas de transferencia (hopper)

\section{CONCLUSIONES}

1. El estudio de expansión a 140,000 tpd en la mina es altamente viable considerando el transporte del mineral a la planta concentradora mediante chancado a la salida del tajo y acarreo por fajas transportadoras.

2. La implementación de un sistema de chancado dentro del tajo se torna atractiva a partir del año 2020 , debido a que los ahorros de capital y costo operativo de los volquetes en los primeros 12 años (2008-2019) no son suficientes como para pagar la inversión del sistema chancado/fajas dentro del tajo.

3. Según el análisis de costos comparativos (costos operativos), el ahorro es el siguiente:

Faja - Volquete $=0,135 \mathrm{\$} / \mathrm{t}$.

Faja - Trenes $=0,066 \$ / \mathrm{t}$.

4. Según la evaluación económica, se tiene:

$\mathrm{TIR}=183 \%$

VPN. TD a $12 \%=6,74 \mathrm{M} \$$

VPN. TD a $15 \%=5,95 \mathrm{MS}$

Periodo de recuperación $=1,5$ años

\section{BIBLIOGRAFÍA}

Arce Q. G. Mineria a Cielo Abierto en Inti Raymi Oruro Bolivia

Buró. A. Aspectos generales de los Yacimientos en Skarn. Horwar University U.S.A.

Bustillos R. Manual de Evaluación y diseño de Explotación Minera. Ed. Madrid - España

Orderique, Edgardo / Pérez, Wilbert / Bernabé, Heller, Plan de Desarrollo Fase 2 Estudio de Expansión Mina Cuajone a 140,000 TPD, Cuajone, 2002

Mintec/Ingeniería Mina Cuajone SPCC, Expansión con chancadora/faja vs. soló volquetes dentro del tajo, Cuajone, 2002. 